Broman-Fulks, J. J., McCloskey, M. S., \& Berman, M. E. (2007). Anxiety sensitivity and retaliatory aggressive behavior in research volunteers. Aggressive Behavior, 33(2): 137-144. (Mar 2007) Published by Wiley (ISSN: 10982337). The definitive version is available at www3.interscience.wiley.com.

\title{
Anxiety Sensitivity and Retaliatory Aggressive Behavior in Research Volunteers
}

\author{
Joshua J. Broman-Fulks, Michael S. McCloskey, and Mitchell E. Berman
}

\begin{abstract}
An important focus of recent aggression research has been to identify personality variables that influence the expression of aggression. One such variable may be anxiety sensitivity (AS). Individuals high in AS fear unpleasant anxiety-related physiological sensations and perhaps physiological arousal in general. Accordingly, people high in AS are motivated to avoid situations that produce these sensations. With respect to aggressive encounters, an intense attack by an opponent involves significant physiological arousal. High anxiety-sensitive individuals may therefore attempt to decrease the intensity of the interaction by responding in a non-aggressive or conciliatory manner. To test this possibility, 112 community volunteers completed a measure of AS and a well-validated laboratory task designed to assess aggressive behavior. As predicted, an inverse relation between AS and extreme aggressive responding was found. Specifically, as AS increases, retaliatory aggression is less likely after intense attack by an opponent.
\end{abstract}


Aggression researchers have long been interested in how personality influences the expression of aggression [e.g., Berman et al., 1998; Giancola and Chermack, 1998]. Although researchers have been able to identify personality variables that are reliably associated with increased aggressive responding, such as Type A behavior [Berman et al., 1993], prejudice [Genthner and Taylor, 1973], and trait anger [Giancola, 2002], few published studies have examined personality styles or traits that reduce the likelihood of aggression. One personality trait that has been shown to be inversely related to aggressive responding is empathy [Giancola, 2003]. This relationship is not surprising, given that individuals high in empathy are sensitive to the misfortunes of others. More generally, personality traits that are potentially associated with discomfort during an aggressive encounter may be expected to be associated with a lower propensity to retaliate after attack or provocation. Anxiety sensitivity (AS) may be one such personality variable.

AS is characterized by a fear of anxiety-related sensations, arising from the belief that these sensations can have harmful consequences [Reiss and McNally, 1985]. Anxiety-sensitive individuals tend to misinterpret and catastrophize relatively benign anxiety-related physiological sensations, such as believing that minor heart palpitations indicate an imminent heart attack. Research has also demonstrated that individuals with high AS tend to respond more fearfully to physiological stimulation [McNally and Eke, 1996; Messenger and Shean, 1998; Sturges et al., 1998; Telch et al., 1996]. For these reasons, high AS individuals are thought to avoid stimuli that evoke intense physiological sensations [McNally, 1996; Taylor and Federoff, 1999]. Evidence supporting this notion comes from three sources-studies on the relation between AS and (1) drug preferences, (2) exercise, and (3) analgesic use.

Results of studies on the relation between AS and drug preferences indicate that high-AS individuals prefer sedatives over stimulants [Norton et al., 1997; Stewart et al., 1995]. However, there is no compelling evidence that anxiety-sensitive individuals avoid arousal-inducing stimulant substances. For example, McWilliams and Asmundson [2001] failed to find an inverse association between AS and stimulant use (i.e., nicotine and caffeine). The amount of caffeine 
consumption reported by participants in this study, however, might have been too little to produce levels of physiological arousal that even high-AS individuals would consider unpleasant. In addition, the expected association between nicotine consumption and AS may have been confounded by the fact that many people report smoking to have a relaxing effect [Scheitrum and Akillas, 2002].

Anxiety-sensitivity studies of exercise and analgesic use have provided qualified support for the notion that high-AS individuals are less likely to engage in behaviors that produce intense physiological sensations. For example, researchers have reported a negative correlation between AS and exercise frequency [Broman-Fulks et al., 2004; McWilliams and Asmundson, 2001]. AS is also associated with pain avoidance. For example, Asmundson and Norton [1995] found that AS was positively related to the use of analgesic medications in chronic pain patients, supporting the notion that high-AS chronic pain patients may be more predisposed to increase their usage of analgesic medications as a form of pain escape or avoidance compared to low-AS patients.

Taken together, these results suggest that people who are high anxiety sensitive are more likely to avoid intense physiological sensations. Indeed, Cox [1996] has suggested that, "AS may be part of a broader set of beliefs about the harmfulness of unusual or strong internal sensations that may or may not be identified as anxiety symptoms" (p. 365). Thus, it may be the case that high-AS individuals actively avoid intense physiological sensations, whether caused by anxiety or other physical or situational events. Studies to date, however, have relied on retrospective, self-reports of avoidance behaviors, and none has examined if AS increases avoidance of aggressive interactions or behavior. Indeed, intense physiological sensations and aggression go hand in hand [Zillman, 1988]. Specifically, physiological arousal increases after attack, which in turn can elicit retaliatory aggressive behavior in the target of attack [Taylor, 1967].

Not all individuals, however, respond to attack in the same fashion. Given that high AS is associated with avoidance of unpleasant physiological sensations, it is possible that high-AS individuals behave less aggressively in response to provocation or 
attack to both minimize arousal and the possibility of subsequent attack. Accordingly, the purpose of this study was to examine the relation between AS and retaliatory aggression using a well-established laboratory measure of aggression. We hypothesize that AS will be inversely associated with aggressive responding, especially after attack by a highly provocative opponent.

\section{METHOD}

\section{Participants}

Participants were 74 men and 38 women $(\mathrm{N}=112)$ ages $18-48$ years $(M=24.75 ; S=56.90)$. The sample was predominately Caucasian $(72 \%)$ and African American (23\%), never married (83\%), and relatively well-educated (93\% had at least some college education). Median income was in the $\$ 12,500-14,999$ range. Volunteers were recruited via community postings for a study on "psychomotor skills." Participants received financial compensation for their time. The consent process and procedures were reviewed and approved by the Institutional Review Board for the Protection of Human Subjects at the University of Southern Mississippi.

\section{AS and Trait Anxiety}

The Anxiety Sensitivity Index [ASI; Peterson and Reiss, 1992] is a widely used measure of AS and is believed to assess general AS (total score) and three components of AS: (1) fear of physical symptoms of anxiety, (2) fear of cognitions associated with anxiety attacks, and (3) fear of publicly observable symptoms of anxiety [Zinbarg et al., 1997]. For this study, ASI total scores were used. The psychometric properties of the ASI are well-established and are only briefly mentioned here. The ASI has relatively good stability across 2 weeks, with a test-retest correlation of .75, and good internal consistency, with alpha scores ranging between .82 and .91 . The ASI has also been shown to possess adequate criterion-related validity, and discriminates individuals with anxiety disorders from non-cases

[Reiss et al., 1986]. 
Controversy has arisen over whether AS can be differentiated from trait anxiety [e.g., Lilienfeld, 1996; Lilienfeld et al., 1989; McNally, 1989, 1996]. Although some maintain that AS is a lower-order facet of a higher-order trait anxiety dimension [Lilienfeld, 1996], research has generally supported the notion that AS is an empirically and conceptually distinct construct [e.g., Rapee and Medoro, 1994]. Whereas trait anxiety refers to a general tendency to respond fearfully to stressors, AS denotes a specific fear of anxiety-related symptoms [McNally, 1999]. To determine if generalized anxiety and AS have similar relationships with retaliatory aggression, or if this relationship is limited to AS, which is more directly related to fear of anxietyrelated symptoms, trait anxiety was also included as an independent variable, and the separate and combined effects of AS and trait anxiety on aggressive responding were examined. Trait anxiety was assessed using the State-Trait Anxiety ScaleTrait [STAI-T; Spielberger et al., 1983]. The STAI-T is a 20 -item self-report measure designed to assess general anxiety proneness. Participants rate the frequency of their anxious feelings on a four-point Likert-type scale from 1 ("almost never") to 4 ("almost always"). Higher scores on the STAI-T indicate increasing levels of trait anxiety. The psychometric properties of the STAI-T are welldocumented and reported in detail elsewhere [Spielberger, 1983].

\section{Taylor Aggression Paradigm (TAP)}

The TAP is a classic laboratory measure of aggressive behavior [Taylor, 1967]. In the TAP, participants are led to believe that they are competing with another participant (a fictitious opponent) in a 28-trial competitive reaction-time game. Before each trial, the participant chooses a shock from a range of intensities to administer to the opponent should the participant "win" (have a faster reaction time) on a given trial. On losing trials, the participant receives a shock ostensibly pre-set by the opponent before the trial. The shock level selected by the fictitious opponent on both winning and losing trials is communicated to the participant via a series of lights labeled 0 (no shock selected), 1-10, and 20 (denoting increasingly intense shock levels). Aggression is operationally defined as the 
level of shock the participant sets for the opponent on each trial.

A rich literature supports the validity of the inferences that can be drawn from the TAP and related laboratory measures of physical aggressive behavior [Anderson and Bushman, 1997; Giancola and Chermack, 1998; McCloskey and Berman, 2003], and the construct validity for the TAP has been repeatedly shown via correlations with selfreport and interview measures of trait aggressiveness [e.g., Berman et al., 2002; Bernstein et al., 1987; Giancola and Zeichner, 1995]. Furthermore, when participants are divided into groups based on aggression history, high-aggression groups respond more aggressively on the TAP when unprovoked, and even more so when provoked [e.g., Bushman et al., 2001]. The paradigm also discriminates groups of participants theoretically expected to evidence elevated levels of aggression, such as psychopathic individuals [Dengerink, 1971], prejudiced persons [Genthner and Taylor, 1973], and individuals with high levels of endogenous testosterone [Berman et al., 1993].

\section{Procedure}

The ASI and STAI-T were embedded in a battery of paper-and-pencil measures. The other measures were administered for separate purposes and are reported elsewhere. After completing the paper-and-pencil measures, the participant was seated in front of the TAP apparatus [for a description of the apparatus see Berman and Walley, 2003]. Two stainless-steel fingertip electrodes with surface areas of 10_19mm were placed on the ventral portion of the index and middle fingers of the non-dominant hand without conductant and held in place with Velcro straps. The experimenter informed the participant that he or she would be competing in a task against another (fictitious) "subject," who was in another room in the research suite. It was explained the two "subjects" would not be allowed to see each other at any point during the experiment as a "protective measure." This fictitious opponent was always the same gender as the participant. The experimenter then excused himself ostensibly to prepare the fictitious opponent for the experiment. After a short delay, an upper shock discomfort threshold was determined by administering increasingly 
intense shocks at 0.10 milliamperes intervals until the participant reported that the shock was "very unpleasant." To increase the credibility of the experimental situation, this procedure was repeated with the other "subject" (an audiotape of a confederate), and overheard by the participant. After the threshold determination, task instructions were provided via intercom to both "subjects," indicating that the purpose of the task was to see which subject could lift a finger off a reaction-time key the fastest. The participant, and ostensibly his or her opponent, selected from a shock form 0 through 10 or 20 by pressing one of 12 buttons on the bottom of the console. The slower person on each trial would receive the shock chosen by his or her opponent before that trial. The 10 shock was equivalent to the shock level judged very unpleasant. The 9 was set at $95 \%$ of this maximum; 8 at $90 \% ; 7$ at $85 \%$, and so forth. We told participants that the 20 shock would administer a "severe" shock, twice the intensity of the 10. Accordingly, a 20-shock selected by the participant indicates extreme aggression towards the opponent. The "subjects" were told that if they selected a 0 , no shock would be administered to "the other person" on losing trials (a non-aggressive response option included to increase the ecological validity of the task).

Participants next completed 28 trials consisting of an initial trial, followed by two blocks of 13 trials. Blocks were separated by a trial of intermediate intensity to smooth the transition between blocks. The shock ostensibly set by the fictitious opponent before each trial was communicated to the participant via the lights labeled 0 and 1-10 through 20. Provocation was manipulated by increasing the opponent's average shock setting from Block 1 (mean shock54.0) to Block 2 (mean shock58.5). Block 2 also included one trial (not included in the determination of the Block 2 average above) in which the opponent selects a 20 -shock for the participant. This provides a clearly aggressive attack by the opponent. In the one instance the fictitious opponent selects a 20 , the participant does not receive the shock because she or he "wins" the trial. We provided no other information about the role of shock in the task. The participant lost (received the opponent shock) on half the trials, with the frequency of wins and losses preprogrammed by the experimenter. After the TAP, participants were debriefed to determine if he or she accepted the social conditions of the task; that is, if he 
or she believed that they were interacting with another participant and if they were unaware that the purpose of the study was to examine aggressive behavior.

\section{RESULTS}

Analyses were conducted 2-tailed at the .05 level of significance. Before analyzing the data, 20 shocks were re-coded as 11 to minimize the influence of 20 shock selections on mean shock calculations [e.g., Myerscough and Taylor, 1985].

\section{Preliminary Analyses}

Cover task. Three participants indicated that they believed we were studying aggression, and two other participants reported that they did not believe the opponent was real. These individuals were excluded from all analyses. Thus, the final sample $(\mathrm{N}=112)$ consisted of participants who accepted the social conditions of the TAP and understood that the shocks chosen by their opponent would be administered to him or her on losing trials. Demographic variables. To ensure that the association between both AS and trait anxiety and aggressive responding was not due to other demographic variables that happened to covary with AS or trait anxiety, zero-order correlations were calculated between scores on both the ASI (M=15.90, $\mathrm{S}=59.63)$ and STAI-T $(\mathrm{M}=33.93, \mathrm{~S}=511.05)$ and age, education, and ethnicity. No relation between any of the demographic variables and either AS or trait anxiety was found. Thus, these variables were not included in the main analyses. Gender differences for age, race, and education level were also assessed. Male and female participants did not differ on age, $\mathrm{t}(108)<1$, education level, $X^{2}(6)=2.30, P=.89$, or race, $X^{2}(5)=2.98, P=.70$.

Study variables. Scores on the ASI and the STAI-T were correlated, $r(112)=.40, P<.001$, sharing approximately $16 \%$ of their variance. Independent samples t-tests were used to assess potential gender differences for both anxiety sensitivity (ASI) and trait anxiety (STAI-T). Results revealed a significant gender difference for trait anxiety, $\mathrm{t}(110)=2.04, \mathrm{P}=.04$, with women $(M=36.86, S D=10.25)$ reporting more trait anxiety 
than men $(M=32.41, S D=12.07)$. No gender

difference was found for AS.

\section{Regression Analyses of Shock Setting Behavior}

Two indices of aggressive behavior, mean shock selection, and use of the 20 shock, were used as dependent variables in separate sets of regression analyses. We expected AS to be inversely related to both indices of aggressive behavior, but primarily in the second block under high levels of provocation. In addition, we attempted to determine if gender moderates this relationship, and if AS and generalized anxiety have similar relationships with retaliatory aggression. Thus, the individual and combined effects of AS, trait anxiety, gender, and provocation on aggressive and non-aggressive responding were examined.

Because both trait anxiety and AS are continuous in nature, regression analyses were employed. ASI and STAI-T scores were z-transformed to center the variables [Aiken and West, 1991; Jaccard et al., 1990]. Gender was dummy coded with men51 and women $=-1$. Interactions terms were created by multiplying the relevant first-order variables [Jaccard et al., 1990]. For this model, unstandardized b weights were interpreted, as standardized beta weights provide inaccurate values for interaction terms. All main effects, as well as 2-way and 3-way interactions, were entered into the regression models simultaneously. This resulted in a full model comprising seven variables. Significant interactions between continuous variables were evaluated by plotting the effect of one predictor variable on the dependent variable (DV) at one SD above the mean, the mean, and one SD below the mean of the second predictor variable and testing to determine whether the slopes of the simple regression lines differed from zero [Jaccard et al., 1990].

In order to use regression analyses in a repeated measures design (the two levels of provocation), a sum/difference regression method was employed in which two DVs are examined in separate regression models. The sum of the two provocation blocks (DV1) was used to examine between-subjects effects independent of provocation, and the difference between low and high provocation trials 
(DV25high provocation - low provocation) was

used to examine the interaction between the within subjects

provocation effect and the between-subjects

variables. This approach allows for the exploration

of all interactions without the need to dichotomize

continuous data [Judd et al., 2001].

Mean shock aggression. To determine how average shock levels vary as a function of AS, trait anxiety, and gender, two regression models were created. The first regression model examined between-subjects effects independent of provocation. The full model was significant $F(7,104)=2.45$, $P=.023 ; R^{2}=.14$. A significant effect of gender $(b=0.77, S E=0.23 t=3.27, P=.001)$ was noted, with men $(M=5.52, S=52.26)$ setting higher average shocks compared to women $(M=4.14$, $\mathrm{SD}=2.07$ ). No other significant effects emerged from this analysis. The second regression examined the association between AS, trait anxiety, and gender on provocation-based changes in mean aggressive responding. The full model was not significant, $F(7,104)=-1.95, P=.069 ; R^{2}=.12$.

Extreme shock aggression. Two regression analyses were again performed to evaluate the effect of AS, trait anxiety, and gender on extreme (20 shock) aggression, defined as the number of 20 shocks selected by the participant to administer to the opponent. The first regression analysis examined the role of these variables independent of provocation. The full model was significant, $F(7,104)=4.23$, $P<.001 ; R^{2}=.22$. AS $(b=-1.20, S E=0.42$, t5_2.85, $P=.005)$ and gender $(b=1.14$, $\mathrm{SE}=0.44, \mathrm{t}=2.73, \mathrm{P}=.007$ ) were the only significant effects in the model. Men $(M=2.57$, $\mathrm{SD}=4.95)$ set more extreme shocks than women $(\mathrm{M}=0.53, \mathrm{~S}=51.29)$. As shown in Figure 1, as AS scores increase, use of the 20 option tends to decrease in both provocation conditions. The second regression examined how AS, trait anxiety, and gender interact with increasing provocation on use of the extreme aggressive response. Although the full model was not significant $F(7,104)=1.58, P=.15 ; R^{2}=.10$, a significant $A S x$ provocation interaction emerged $(b=-0.42$, $\mathrm{SE}=0.20 \mathrm{t}=-2.09, \mathrm{P}<.05)$. As can be seen if Figure 1 , participants with the lowest AS scores were most reactive to provocation; that is, lower AS scores were associated with higher counterattack with the extreme 20 response when provoked, and these high vs. 
low-provocation differences in counterattack

decreased as AS scores increased above the mean.

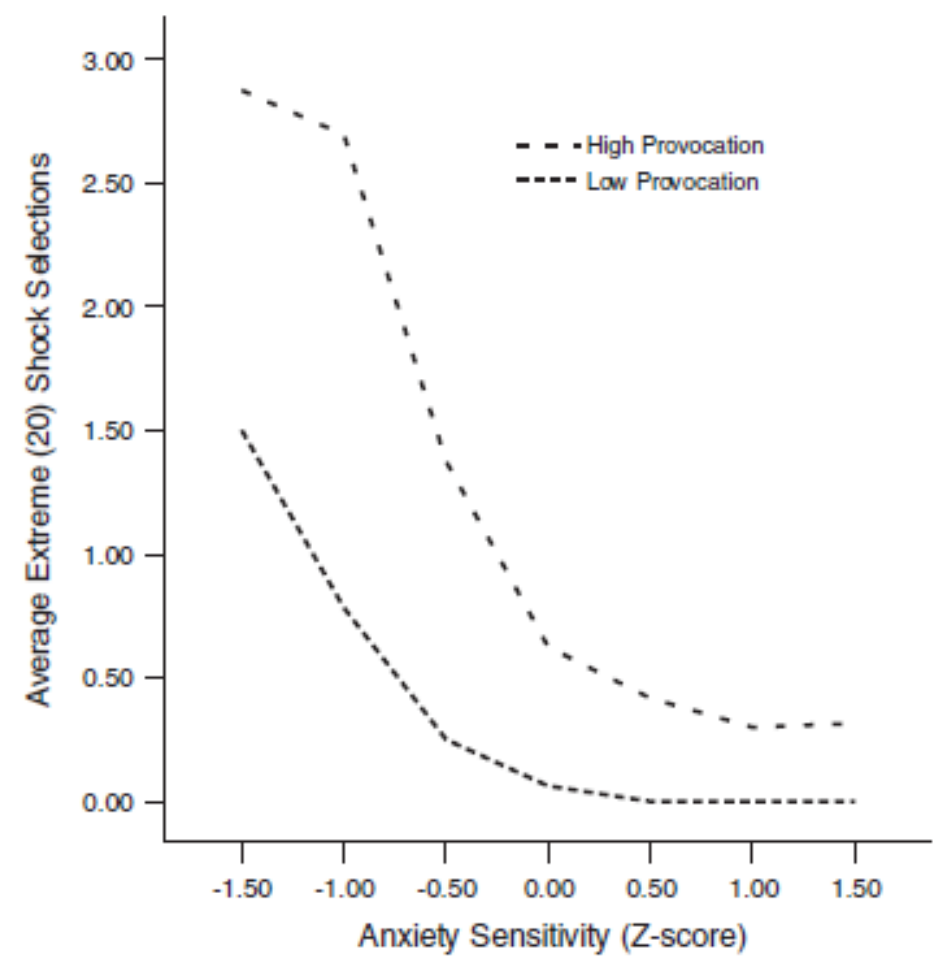

Fig. 1. Mean number of extreme (20) shock selections administered as a function of anxiety sensitivity and provocation.

\section{Use of the 0 Response}

The correlation between the two "extreme" response options, the 20 and 0 , was non-significant, $r=-.098, P=.30$. Thus, the 0 response option may provide independent information about the relation between AS and a clearly non-aggressive response. Accordingly, a regression analysis was conducted for AS and provocation with the number of 0 shocks the participant selected for his or her opponent as the dependent variable. No main effects or interactions for AS emerged from this analysis.

\section{DISCUSSION}

The purpose of the present study was to examine the relation between AS and retaliatory aggressive 
behavior. In his initial conceptualization of AS, Reiss [1991] proposed that persons with high AS fear the experience of unpleasant anxiety-related physiological sensations. Thus, they are motivated to avoid stimuli that are likely to create anxiety [Reiss, 1991]. Theorists have thus suggested that negative associations should exist between AS and physiologically arousing substances, situations, and behaviors [see McNally, 1996]. It was therefore predicted that AS would be inversely related to retaliatory aggressive responding, a situation that involves physiological arousal.

Results were consistent with this prediction, but only for extreme aggressive behavior. Specifically, AS did not predict mean aggression levels, but AS was inversely associated with the use of an extreme aggressive response, especially after an intense attack by an opponent. In other words, even though participants observed the opponent select a highly painful shock for them to receive, those with higher levels of AS were less likely to respond in kind.

Given the different pattern of results for mean and extreme aggression, it may be that behaviors that produce mild increases in physiological sensations are not avoided by high-AS individuals, but behaviors that generate more intense sensations are avoided. In addition, the pattern for extreme aggression supports the notion that AS has a small relation with aggression when provocation is minimal, but clear conciliatory behavior is expressed by high-AS people when attacked.

Although our AS arousal-avoidance hypothesis is plausible and appears to be supported, it is possible that the relation between AS and aggressive responding merely reflects a more general propensity for trait-anxious people to avoid retaliatory aggression. However, no evidence was found to support this position. Results indicated that trait anxiety did not predict aggressive responding, including extreme displays of aggression.

One unique feature of the present study was the inclusion of an unequivocally non-aggressive response option. The vast majority of TAPbased aggression research forces participants to administer some level of shock. The fact that the present study included a response that did not deliver any shock (the 0) provides the opportunity to examine AS in the context of a true 
"non-aggressive" response. Results indicated that AS was not associated with non-aggressive responding. Thus, it appears that AS does not prevent one from entering an aggressive encounter but that high-AS individuals are less likely to display escalating levels of aggression in response to high provocation.

AS is a multifaceted construct related to a variety of other individual difference variables. Future research may wish to evaluate and control for some of these variables that may serve to mediate or moderate the relation between AS and various forms of arousal avoidance. For example, it is possible that psychopathy traits, such as callousness or guiltlessness, may play a role in the AS-aggression association. Previous research has shown a negative association between AS and the core affective deficits of psychopathy [Lilienfeld and Penna, 2001]. Thus, it may be that individuals with higher levels of AS are more empathetic or guilt-prone, making them less likely to retaliate against an aggressor than people with little capacity for empathy or guilt. It is also possible that these findings were influenced by a positive association between AS and social desirability, with more highly anxiety-sensitive individuals being more sensitive to negative evaluation. Indeed, one component of the AS construct is a fear of negative evaluation associated with the display of anxiety symptoms [Zinbarg et al., 1997]. Consideration of such variables may help explain the processes responsible for the AS-aggression link observed here.

A significant body of literature exists to support the notion that negative affectivity and aggression are positively associated [see Berkowitz, 1990]. In fact, a wide array of unpleasant emotions, such as sadness, depression, and anxiety, have been linked to rises in aggression and anger. Research has also indicated that AS is positively associated with negative affectivity [Lilienfeld, 1997]. Thus, although one may have reasonably predicted a positive association between aggressive behavior and AS, the opposite appears to be true. It is possible that the unpleasant emotions associated with increased autonomic arousal in high-AS individuals are superseded by their drive to avoid the prolongation or intensification of such symptoms. Additional research will be needed to 
further clarify the direct and indirect relations between AS, negative emotionality, and aggressive behavior.

The relation between aggression and gender is well-documented. In general, men tend to behave more aggressively than women, a finding which may be most clearly evidenced by violent crime statistics across cultures. In addition to being more apt to commit crimes such as murder, aggravated assault, and armed robbery, men are also more likely to react aggressively to a variety of interpersonal conflicts [Reinisch and Sanders, 1986]. Laboratory studies of gender differences in aggressive responding have generally supported these naturalistic findings [Zeichner et al., 2003]. The results of the present study are consistent with those of previous reports. On average, men selected higher levels of shock and more frequent extreme shocks (i.e., 20 shocks) compared to their female counterparts. Several potential explanations have been proposed to account for the effects of gender on aggression. First, biological factors, such as testosterone levels, may play a role in the production of an aggressive response [Berman et al., 1993; Maccoby and Jacklin, 1980]. Second, a variety of social and cultural factors may combine to produce gender differences. For example, women may be more inhibited about aggressing than men, and experience more anxiety, fear, and guilt after aggressing [Eagly and Steffen, 1986]. If this is the case, we would expect anxietysensitive women to be the least likely to engage in an aggressive response in comparison with other females and their male counterparts. Because of the relatively few number of participants who scored either extremely high or low on the ASI, the power to detect such effects may have been limited. Future research may wish to address this issue by comparing retaliatory aggression in men and women at various points on the AS continuum.

Cox [1996] has suggested that AS may be a component of a more general set of fears of strange or intense somatic sensations that may or may not be associated with anxiety. Evidence from the present study and recent research indicating that anxiety-sensitive persons tend to avoid physiological arousal associated with physical exercise and pain appear to support this possibility. When the items of the ASI are independently examined, only one appears to specifically assess fear of anxiety-related 
sensations, whereas the remaining items assess fear of sensations that are not anxiety-specific [Cox, 1996]. Thus, it is recommended that future research attempt to resolve the issue of whether a more general fear of autonomic arousal exists; and if so, whether it provides predictive information beyond that supplied by AS's more specific fear of anxiety sensations.

\section{ACKNOWLEDGMENTS}

This study was supported in part by a grant from the National Institute of Mental Health to Mitchell E. Berman (MH57133).

\section{REFERENCES}

Aiken L, West S. 1991. "Multiple Regression: Testing and Interpreting Interactions," Newbury Park, CA: Sage Publications. Anderson CA, Bushman BJ. 1997. External validity of "trivial" experiments: The case of laboratory aggression. Rev Gen Psychol 1:19-41.

Asmundson GJG, Norton GR. 1995. Anxiety sensitivity in patients with physically unexplained chronic back pain: A preliminary report. Behav Res Ther 33:771-777.

Berkowitz L. 1990. On the formation and regulation of anger and aggression: A cognitive-neoassociationistic analysis. Am Psychol 45:494-503.

Berman ME, Walley JC. 2003. Imitation of self-aggressive behavior: An experimental test of the contagion hypothesis. J Appl Soc Psychol 5:1036-1057.

Berman ME, Gladue B, Taylor S. 1993. The effects of hormones, Type A behavior pattern, and provocation on aggression in men. Motivat Emot 17:125-138.

Berman ME, Fallon A, Coccaro EF. 1998. The relationship between personality psychopathology and aggressive behavior in research volunteers. J Abnorm Psychol 107:651-658.

Berman ME, McCloskey M, Coccaro EF, Greer T. 2002. Gender, testosterone, and human aggression. Poster presented at the 2002 Annual Meeting of the American Psychological Association, Chicago, IL. 
Bernstein S, Richardson D, Hammock G. 1987. Convergent and discriminant validity of the Taylor and Buss measures of physical aggression. Aggr Behav 13:15-24.

Broman-Fulks JJ, Broman-Fulks JD, Sellinger J, Berman ME. 2004. Anxiety sensitivity and arousal avoidance. Poster presented at the Annual Meeting of the Southeastern Psychological Association, Atlanta, GA.

Bushman BJ, Baumeister RF, Phillips CM. 2001. Do people aggress to improve their mood? Catharsis beliefs, affect regulation opportunity, and aggressive responding. J Pers Soc Psychol 81: 17-32.

Cox BJ. 1996. The nature and assessment of catastrophic thoughts in panic disorder. Behav Res Ther 34:363-374.

Dengerink HA. 1971. Anxiety, aggression and physiological arousal. J Exp Res Pers 5:223-232.

Eagly AH, Steffen VJ. 1986. Gender and aggressive behavior: A meta-analytic review of the social psychological literature.

Psychol Bull 100:309-330.

Genthner RW, Taylor SP. 1973. Physical aggression as a function of racial prejudice and the race of target. J Pers Soc Psychol 27:

207-210.

Giancola PR. 2002. The influence of trait anger on the alcoholaggression relation in men and women. Alcoholism: Clin Exp Res 26:1350-1358.

Giancola PR. 2003. The moderating effects of dispositional empathy on alcohol-related aggression in men and women. J Abnorm Psychol 112:275-281.

Giancola PR, Chermack ST. 1998. Construct validity of laboratory aggression paradigms: A response to Tedeschi and Quigley 1996. Aggr Violent Behav 3:237-253.

Giancola PR, Zeichner A. 1995. Construct validity of a competitive reaction time aggression paradigm. Aggr Behav 21:

199-204.

Jaccard J, Turrisi R, Wan C. 1990. "Interaction Effects in Multiple Regression," Newbury Park, CA: Sage Publications.

Judd C, Kenny D, McClelland H. 2001. Estimating and testing mediation and moderation in within subject designs. Psychol Meth 6:115-134. 
Lilienfeld SO. 1996. Anxiety sensitivity is not distinct from trait anxiety. In: Rapee RM (ed): "Current Controversies in the Anxiety Disorders," New York: Guilford, pp 228-244.

Lilienfeld SO. 1997. The relation of anxiety sensitivity to higher and lower order personality dimensions: implications for the etiology of panic attacks. J Abnorm Psychol 106:539-544.

Lilienfeld SO, Penna S. 2001. Anxiety sensitivity: Relations to psychopathy, DSM-IV personality disorder features, and personality traits. J Anxiety Disorders 15:367-393.

Lilienfeld SO, Jacob RG, Turner SM. 1989. Comment of Holloway and McNally's 1987. "Effects of anxiety sensitivity on the response to hyperventilation." J Abnorm Psychol 98: 100-102.

Maccoby EE, Jacklin CN. 1980. Sex differences in aggression: A rejoinder and reprise. Child Dev 51:964-980.

McCloskey M, Berman ME. 2003. Laboratory measures of aggression: the Taylor Aggression Paradigm. In: Coccaro EF (ed): "Aggression: Assessment and Treatment into the 21st Century," New York: Marcel Dekker, pp 195-214.

McNally RJ. 1989. Is anxiety sensitivity distinguishable from trait anxiety? A reply to Lilienfeld, Jacob, and Turner 1989. J Abnorm Psychol 98:193-194.

McNally RJ. 1996. Anxiety sensitivity is distinguishable from trait anxiety. In: Rapee RM (ed): "Current Controversies in the Anxiety Disorders," New York: The Guilford Press, pp 214-227.

McNally RJ. 1999. Theoretical approaches to the fear of anxiety. In: Taylor S (ed): "Anxiety Sensitivity: Theory, Research, and Treatment of the Fear of Anxiety," New Jersey: Lawrence Erlbaum Associates, pp 3-16.

McNally RJ, Eke M. 1996. Anxiety sensitivity, suffocation fear, and breath-holding duration as predictors of response to carbon dioxide challenge. J Abnorm Psychol 105:146-149.

McWilliams LA, Asmundson GJG. 2001. Is there a negative association between anxiety sensitivity and arousal-increasing substances and activities? J Anxiety Disord 15:161-170.

Messenger C, Shean G. 1998. The effects of anxiety sensitivity and history of panic on reactions to stressors in a non-clinical sample. J Behav Ther Exp Psychiatry 29:279-288. 
Myerscough R, Taylor SP. 1985. The effects of marijuana on human physical aggression. J Pers Soc Psychol 49:1541-1546.

Norton GR, Rockman GE, Ediger J, Pepe CP, Goldberg S, Cox B, Asmundson GJG. 1997. Anxiety sensitivity and drug choice in individuals seeking treatment for substance abuse. Behav Res Ther 35:859-862.

Peterson RA, Reiss S. 1992. "Anxiety Sensitivity Index Revised Manual," Worthington, OH: International Diagnostic Systems Publishing Corporation.

Rapee RM, Medoro L. 1994. Fear of physical sensations and trait anxiety as mediators of the response to hyperventilation in nonclinical subjects. J Abnorm Psychol 103:693-699.

Reinisch JM, Sanders SA. 1986. A test of sex differences in aggressive response to hypothetical conflict situations. J Pers Soc Psychol 50:1045-1049.

Reiss S. 1991. Expectancy model of fear, anxiety, and panic. Clin Psychol Rev 11:201-214.

Reiss S, McNally RJ. 1985. The expectancy model of fear. In: Reiss S, Bootzin RR (eds): "Theoretical Issues in Behavior Therapy," London: Academic Press, pp 107-121.

Reiss S, Peterson RA, Gursky DM, McNally RJ. 1986. Anxiety sensitivity, anxiety frequency, and the prediction of fearfulness. Behav Res Ther 24:1-8.

Scheitrum RR, Akillas E. 2002. Effects of personality style, anxiety, and depression on reported reasons for smoking. J Appl Biobehav Res 7:57-64.

Spielberger CD. 1983. "Manual for the State-Trait Anxiety Inventory, Revised Edition," Palo Alto, CA: Consulting Psychologist Press.

Spielberger CD, Gorsuch RL, Lushene RE, Jacobs GA. 1983.

"State-Trait Anxiety Inventory for Adults," Palo Alto, CA:

Consulting Psychologists Press.

Stewart SH, Peterson JB, Pihl RO. 1995. Anxiety sensitivity and selfreported alcohol consumption rates in university women. J Anxiety Disord 9:283-292.

Sturges LV, Goetsch VL, Ridley J, Whittal M. 1998. Anxiety sensitivity and response to hyperventilation challenge: Physiologic arousal, interoceptive acuity, and subjective distress.

J Anxiety Disord 12:103-115. 
Taylor SP. 1967. Aggressive behavior and physiological arousal as a function of provocation and the tendency to inhibit aggression.

J Pers 35:297-310.

Taylor S, Federoff IC. 1999. The expectancy theory of fear, anxiety, and panic: A conceptual and empirical analysis. In: Taylor S (ed):

"Anxiety Sensitivity: Theory, Research, and Treatment of the Fear of Anxiety," New Jersey: Lawrence Erlbaum Associates, pp 17-34.

Telch MJ, Silverman A, Schmidt NB. 1996. Effects of anxiety sensitivity and perceived control on emotional responding to caffeine challenge. J Anxiety Disord 10:21-35.

Zeichner A, Parrot DJ, Frey FC. 2003. Gender differences in laboratory aggression under response choice conditions.

Aggr Behav 29:95-106.

Zillman D. 1988. Cognition-excitation interdependencies in aggressive behavior. Aggr Behav 14:13-24.

Zinbarg RE, Barlow DH, Brown TA. 1997. The hierarchical structure and general factor saturation of the anxiety sensitivity index: Evidence and implications. Psychol Assess 9:277-284. 